\title{
Changing Weight Management Self-efficacy Among Obese Puerto Rican Adults: A Quantitative Study using a Health Coaching Intervention
}

Richard Valentin Ayala

A.T. Still University, rvalentin@atsu.edu

Josh Bernstein

A.T. Still University, jbernstein@atsu.edu

Follow this and additional works at: https://nsuworks.nova.edu/ijahsp

Part of the Health and Physical Education Commons, Health Psychology Commons, Medicine and Health Commons, Other Nutrition Commons, and the Public Health Education and Promotion Commons

\section{Recommended Citation}

Valentin Ayala R, Bernstein J. Changing Weight Management Self-efficacy Among Obese Puerto Rican Adults: A Quantitative Study using a Health Coaching Intervention. The Internet Journal of Allied Health Sciences and Practice. 2020 Jan 01;18(1), Article 9.

This Manuscript is brought to you for free and open access by the College of Health Care Sciences at NSUWorks. It has been accepted for inclusion in Internet Journal of Allied Health Sciences and Practice by an authorized editor of NSUWorks. For more information, please contact nsuworks@nova.edu. 


\title{
Changing Weight Management Self-efficacy Among Obese Puerto Rican Adults: A Quantitative Study using a Health Coaching Intervention
}

\begin{abstract}
Purpose: Obesity and associated healthcare-related issues continue to increase. The prevalence of obesity is on the rise, which has led many health professionals to find ways to improve health interventions. Health coaching can be a viable tool to reduce the obesity epidemic. The purpose of this quantitative study was to investigate the effects of an 8-weekhealth coaching intervention in obese individuals from Puerto Rico and to determine if self-esteem and body image influence weight managementself-efficacy. Method: A pretest-posttest design using a weight management self-efficacy scale helped the researcher evaluate the effectof the coaching sessions. In addition, body image was measured using the 9-figure Body Size Scale and self-esteem was analyzedwith the Rosenberg Self-esteem Scale. Forty Hispanic adult men and women participated in the study. Results: Health coaching had a statistically significant effect on weight management self-efficacy ( $(39)=-6.58, p<.001$ ). Additionally, body image and self-esteem affected weight management self-efficacy and positive health coaching outcomes were significant regardless of positive or negative perceived body image. Conclusions: These findings suggest that body image and selfesteem should be considered when creating programs for obesity and a health coaching program can be effective in increasing weight management self-efficacy.
\end{abstract}

\section{Author Bio(s)}

Richard Valentin, DHEd, EdS, MEd, NBC-HWC, is a Lifestyle Health Coach from San Juan, Puerto Rico. He is also a national board certified health and wellness coach.

Josh Bernstein, $\mathrm{PhD}$, is an associate professor of health education in the College of Graduate Health Studies at A.T. Still University.

\section{Acknowledgements}

The authors appreciate those who voluntarily participated in this study and made it possible. 


\title{
1IJAHSP
}

\section{The Internet Journal of Allied Health Sciences and Practice \\ Dedicated to allied health professional practice and education}

Vol. 18 No. 1 ISSN 1540-580X

\section{Changing Weight Management Self-efficacy among Obese Puerto Rican Adults: A Quantitative Study using a Health Coaching Intervention}

\author{
Richard Valentin Ayala \\ Josh Bernstein \\ A. T. Still University \\ United States
}

\begin{abstract}
Purpose: Obesity and associated healthcare-related issues continue to increase. The prevalence of obesity is on the rise, which has led many health professionals to find ways to improve health interventions. Health coaching can be a viable tool to reduce the obesity epidemic. The purpose of this quantitative study was to investigate the effects of an 8-week health coaching intervention in obese individuals from Puerto Rico and to determine if self-esteem and body image influence weight management self-efficacy. Method: A pretest-posttest design using a weight management self-efficacy scale helped the researcher evaluate the effect of the coaching sessions. In addition, body image was measured using the 9-figure Body Size Scale and self-esteem was analyzed with the Rosenberg Self-esteem Scale. Forty Hispanic adult men and women participated in the study. Results: Health coaching had a statistically significant effect on weight management self-efficacy $(t(39)=-6.58, p<.001)$. Additionally, body image and selfesteem affected weight management self-efficacy and positive health coaching outcomes were significant regardless of positive or negative perceived body image. Conclusions: These findings suggest that body image and self-esteem should be considered when creating programs for obesity and a health coaching program can be effective in increasing weight management self-efficacy.
\end{abstract}

Keywords: body image; obesity; quantitative; self-efficacy; self-esteem. 


\section{INTRODUCTION}

\section{Obesity and Health Coaching}

As obesity rates increase globally, health professionals and researchers are looking for effective ways to prevent and treat obesity. 1,2 The number of chronic health conditions related to obesity are increasing, and health professionals must look for more alternatives to approach overweight and obesity with strategies focused on prevention and behavior modification. ${ }^{3}$ Effective lifestyle initiatives and interventions are needed in Puerto Rico as a result of increases in obesity. Puerto Rico is the smallest island of the Greater Antilles, and is disproportionately affected by the highest rates of cardiovascular risk factors, including diabetes, obesity, and hypertension compared to the Caribbean and Latin American countries. ${ }^{4,5}$

Obesity is a serious health problem in Puerto Rico and in the United States. Obesity in the United States population has increased, and Puerto Rico has presented a similar increase in overweight and obesity. ${ }^{6}$ It is estimated that $28.3 \%$ of the Puerto Rican population is obese, and $37.6 \%$ is overweight, which is the highest percentage of all U.S. territories and is higher than 42 of the 50 states. ${ }^{6}$ Puerto Ricans experience higher incidence and prevalence rates of diabetes, asthma, and cardiovascular diseases, all of which are associated with obesity and are primary causes of mortality. ${ }^{7-10}$ Serious disparities in chronic conditions exist, with a prevalence of $42 \%$ in Puerto Rico vs. $31 \%$ in the US for hypertension; $39 \%$ vs. $36 \%$ for high cholesterol; $16 \%$ vs. $10 \%$ for diabetes; and $9 \%$ vs. $6 \%$ for myocardial infarction or coronary heart disease. ${ }^{5}$ Obesity is a serious public health issue that needs to be addressed in Puerto Rico because it can lead to serious chronic issues and health disparities. ${ }^{11}$ Obesity and overweight in Puerto Rico has contributed to high cholesterol, triglycerides, and overall lipid levels as well as a high prevalence of cardiovascular diseases, metabolic syndrome, and cancers. ${ }^{12}$

Obesity is a complex physical, social, emotional, and psychological issue that benefits from a holistic approach. Our culture equates obesity to laziness and lack of willpower and effort.1,13-15 Obese individuals are stigmatized, at times undermining their weight loss efforts and behavioral changes. Unrealistic pressures and being stigmatized for body image and weight negatively affects their self-esteem, body image, and self-efficacy. Negative beliefs are internalized and bring forth negative reactions. ${ }^{16}$ Obese individuals can be denigrated by stigma of their body size and image. ${ }^{17}$ They often face physical and social pressures, which is why obesity should be examined from a physical and psychosocial point of view to understand the frustration and low adherence to positive health choices. ${ }^{18,19} \mathrm{~A}$ multidimensional approach can help make lifestyle changes more effective, which is why alternative programming needs to be explored. ${ }^{4,20}$ Health coaching seems to be an effective multidimensional approach to improve health and wellbeing. ${ }^{21}$

Health coaching focuses on increasing awareness and empowerment. It motivates and facilitates behavior change from within. ${ }^{22}$ Through a health coaching intervention focused on improving self-efficacy, individuals can analyze willingness to change, barriers, benefits of change, strengths, and beliefs. ${ }^{13,23}$ Traditional health education interventions are common, but there are few coachingspecific interventions with a non-judgmental approach, a clear goal setting process, accountability, and client-centered followups. ${ }^{21}$ Health coaching is a personalized approach focused on the individual as a whole. ${ }^{20}$ Coaching interactions help individuals shift their mindset through open discussions that adapt to their values, priorities, needs, and desires. ${ }^{24}$ Coaching interventions facilitate change by increasing motivation, confidence, understanding, and self-efficacy. 22,25

There is a need to make changes in the role of health professionals to include more personalized treatment strategies that consider the individuals' preferences and psychosocial variables. ${ }^{20}$ Health coaching addresses these needs by helping individuals acquire knowledge, skills, and confidence in their capacities and abilities to reach goals. It addresses education, strategies, selfmanagement, exploration, short and long-term goals, compliance, action plans, and barrier resolution. ${ }^{26}$ Health coaching can be used to enable individuals to take action and find solutions. ${ }^{21}$ It can be effective because there is a strong focus on listening, providing feedback, monitoring, facilitating, and supporting the individuals in the process, which is why it shows great potential in the health and wellness field. 20-22 Ongoing support, accountability, and information empowers individuals to change, find solutions, and use effective strategies for weight management. Coaching provides a personalized, feasible, and comprehensive approach. ${ }^{21}$

Despite the increase in popularity of health coaching, further research is needed to demonstrate its efficacy. ${ }^{22}$ For this reason, this study addresses the practical application of health promotion and education using a health coaching intervention. The researcher focused on developing, implementing, and evaluating a practical health coaching program that can advance research and promote best practices. This research focuses on involving health coaches to improve lifestyles and self-efficacy for weight management. The study can help other researchers and health professionals understand factors that may affect an individual's ability to sustain and maintain a healthy lifestyle; the importance of addressing other psychological and emotional variables before focusing on other areas of health improvement; and the strategy of involving health coaching to improve lifestyles and self-efficacy for weight management. In addition, this study will contribute by focusing on a Hispanic population in Puerto Rico. The use of this weight management health coaching intervention will add insight into non-Caucasian populations. The aim of this study is to conduct an 
8-week health coaching intervention to evaluate its effect on weight management self-efficacy and to analyze the correlations between body image, self-esteem, and self-efficacy in obese adults in Puerto Rico.

\section{Self-Efficacy}

Self-efficacy is a person's belief in their ability, capacity, or capability to organize and perform necessary behaviors to produce desired outcomes. ${ }^{27}$ Bandura's self-efficacy model comes from the social cognitive theory, a widely used theoretical framework for behavioral change interventions. ${ }^{27}$ Self-efficacy, which is a vital part of behavior change, reflects an individual's belief and confidence in their ability to positively address personal challenges and issues. High levels of self-efficacy are associated with commitment to successfully adopt new behaviors in self-management. The behavior, expectation, and outcome can be positive if the individuals have confidence in their ability and desire to improve.28-29 Individuals who want to improve weight and health must believe in their ability and capacity to change. ${ }^{30}$

Self-efficacy should be considered in health-interventions because the individuals must believe that they can change their lifestyle behaviors. It is considered a vital part of behavioral change..$^{20,22,27,30}$ Health coaching can have a positive effect on health behaviors and self-efficacy because individuals are encouraged to take greater responsibility for their health and their abilities to create change. Health coaches increase self-efficacy by empowering the individuals to learn and self-manage their health with a personalized approach. ${ }^{31}$

\section{METHOD}

Participants

A non-randomized convenience sample was recruited using local newspaper advertisements in one county in Bayamon, Puerto Rico. The coaching intervention, including individual and group sessions, were held in a local office space in Toa Baja, Puerto Rico. Demographic characteristics varied regarding sex, age, and employment. The researcher recruited 40 Hispanic adult men and women age 22-64. Information about the initial requirements was included in the newspaper advertisement and was discussed again when participants enrolled in the study. No incentives were offered -- just the benefit of improving health with a professional health coach.

The requirements included a body mass index (BMI) over 30, 18-64 years of age, and willingness to participate actively and voluntarily in the study. The researcher verified all participants complied with study parameters using a demographic questionnaire for age and determining BMl using the standard formula. ${ }^{32}$ An Omron HBF-514C full body sensor weight scale and a measuring stick were used to determine weight and height. Information about the study was given to participants and written informed consent was obtained before enrollment in the intervention. Participants were free to decline participation without consequence at any time before or during the study.

\section{Materials}

Age, gender, marital status, status of employment, body mass index, and weight were measured during the pretest process with a socio-demographic questionnaire. In addition, the researcher used three measurement scales in this study. Weight management self-efficacy was measured using the Nutrition Self-efficacy Scale and the Physical Exercise Self-efficacy Scale, which are both a modified version of the General Self-efficacy Scale developed by Jerusalem and Schwarzer. ${ }^{33}$ The researcher combined both scales to create the Weight Management Self-efficacy Scale (WMSS). Both the Nutrition Self-efficacy Scale (5 items; $a=.87)$ and the Physical Exercise Self-efficacy Scale $(5$ items; $\alpha=.88)$ are reliable and valid. ${ }^{34} \mathrm{~A}$ professional translator converted the WMSS from English to Spanish using cross-cultural forward translation and parallel back-translation procedures. The process was repeated twice and then verified by two professionals in the health field for content validity. ${ }^{35}$

Body image was measured using the 9-figure Body Size Scale (BSS) developed by Stunkard, Sörensen, \& Schulsiger, which is valid and reliable $(\alpha=.82, \alpha=.71)$, and the most widely used body type scale. ${ }^{36-37}$ Self-esteem was analyzed with a Spanish version of the scale developed by Rosenberg called the Rosenberg Self-esteem Scale (RSS), which is a valid and reliable tool that has been translated and tested for internal consistency using Cronbach's alpha. ${ }^{38}$ The values obtained in the test-retest correlation were $\alpha=.85, \alpha=.88$, and $\alpha=.84$. This instrument represents the most common tool used to measure self-esteem. ${ }^{39}$

\section{Procedure}

A pretest/posttest design was used to evaluate changes in weight management self-efficacy and to determine if self-esteem and body image influence weight management self-efficacy. The RSS, the 9-figure BSS, and the WMSS scale were the primary data collection tools for this study. All three data collection tools were administered prior to the first coaching session; the WMSS was administered again in the last individual coaching session to assess the effect of the health coaching intervention on weight management self-efficacy. 
Participants engaged in individual and group coaching sessions once a week for a total of eight weeks. The individual coaching sessions were conducted in week one, four, and eight and the group coaching sessions in week two, three, five, six, and seven (Figure 1). The intervention included five 2-hour educational group sessions along with three individual, face-to-face coaching sessions that lasted around 30 minutes each. All meetings, coaching sessions, and data collection took place in an office space in Toa Baja, Puerto Rico and materials and communications were in Spanish. The researcher had over 11 years of experience as a wellness coach and educator, and served as the wellness coach and principal investigator in the study. The researcher followed all protocols and procedures required by A.T. Still University's Institutional Review Board (IRB) to ensure adherence to all standards for adequate protection and well-being of participants. The Ethics Committee of the institution provided approval.

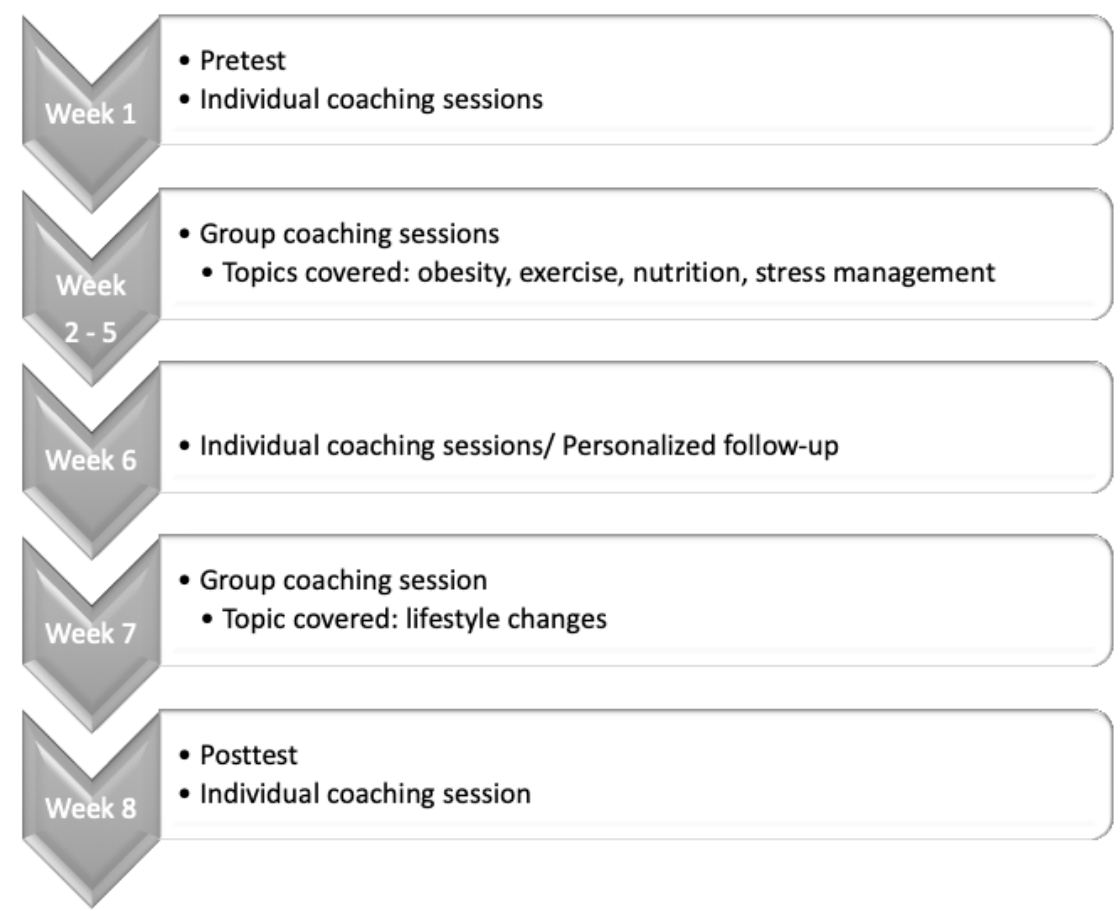

Figure 1. Health Coaching Intervention Timeline

Participants were required to participate in all sessions. Before beginning the group sessions, the researcher met with all participants for an initial coaching session to analyze participant goals, short- and long-term objectives, motivations, obstacles, and to develop action steps. Group sessions were used to address the topics of obesity, exercise, nutrition, stress management, and lifestyle changes. The researcher used the group sessions to discuss ways to improve in those areas, develop practical solutions, and answer all questions. Then, participants were encouraged to write down in their notebooks every possible solution they could put into practice throughout the remainder of the study. The individual coaching sessions were designed to provide follow-up on everything they were putting into practice, feedback, and one-on-one coaching focused on personalized health goals. During the final week of the intervention, the researcher met with all participants and completed the posttest to measure change in self-efficacy.

\section{Data Analysis}

A paired samples t-test was conducted to examine whether the difference between weight management self-efficacy pretest and posttest. Pearson correlation coefficient was used to assess the dependence between the interval-level data collected for selfesteem and self-efficacy. In addition, the researcher assessed the dependence between self-efficacy and body image and the dependence between self-esteem and body image. Frequencies, percentages, and standard deviations were determined. Finally, multiple regression was used to assess if body image and self-esteem influenced weight management self-efficacy. Results were interpreted cautiously using a conservative p-value (0.05). The Statistical Package for the Social Sciences (SPSS) Version 23 was used to analyze the quantitative data. 


\section{RESULTS}

\section{Demographics}

A power analysis was conducted to determine a sufficient sample size using an alpha of 0.05 , a power of 0.80 , a medium effect size ( $\mathrm{dz}=0.5)$, and two tails. Based on the aforementioned delimitations, the minimum desired sample size was 34 . For that reason, a total of 40 Hispanic adult men $(n=15,38 \%)$ and women $(n=25,62 \%)$ between the ages of 22 to 64 , with an average of 46.58 $(S D=10.57)$, participated in the study. Six additional individuals were accepted to ensure data analysis requirements were met and address potential attrition. The observations for $\mathrm{BMI}$ ranged from 30 to 51.9, with an average of $37.16(\mathrm{SD}=6.90)$. Most participants were married $(n=29,72 \%)$ and employed $(n=29,72 \%)$. Frequencies and percentages are presented in Table 1.

Table 1. Frequency Table for Nominal Variables.

$\begin{array}{lll}\text { Variable } & n & \\ \text { Sex } & & 62 \\ \text { Female } & 25 & 38 \\ \text { Male } & 15 & 72 \\ \text { Marital Status } & & 12 \\ \text { Married } & 29 & 15 \\ \text { Divorced } & 5 & 72 \\ \text { Single } & 6 & 28 \\ \text { Employed } & & \end{array}$

Health Coaching and Weight Management Self-Efficacy

A paired samples t-test was conducted to examine whether the difference between weight management self-efficacy pretest and posttest was significantly different from zero after the health coaching intervention. The result of the paired samples t-test was significant, $t(39)=-6.58, p<.001$, suggesting that the true difference in the means of the WMSS pretest and posttest was significantly different from zero. The mean of the pretest $(M=27.30)$ was significantly lower than the mean of the posttest $(M=$ 32.12).

\section{Body Image and Weight Management Self-Efficacy}

A Pearson correlation analysis was conducted among BSS, WMSS pretest, and WMSS posttest. There was a significant negative correlation between BSS and the WMSS pretest $(r=-.50, p=.001)$. The correlation coefficient between the BSS and the WMSS pretest indicated a large effect size. This result suggests that as the BSS increases, the WMSS pretest tends to decrease. The more negatively participants perceived their body image at the beginning of the study, a lower weight management self-efficacy was found, indicating that body image can and did influence weight management self-efficacy without health coaching. No significant correlation was found between the BSS and the WMSS posttest, meaning that their perceived body image did not affect the WMSS posttest increase. The use of coaching strategies, personalized follow-ups and practical support in the health coaching sessions seemed to improve their weight management self-efficacy, despite their perceived body image. Results demonstrated that the health coaching process was effective in improving weight management self-efficacy, closing the gap between participant's perceived body image and self-efficacy.

\section{Self-esteem and Weight Management Self-Efficacy}

A Pearson correlation analysis was conducted among the RSS and the WMSS pretest and posttest. There was a significant positive correlation between the RSS and the WMSS pretest $(r=.35, p=.028)$. The correlation coefficient between the RSS and the WMSS pretest indicated a moderate effect size. This suggests that as the RSS increases, the WMSS pretest tends to increase. There was also a significant positive correlation between the RSS and the WMSS posttest $(r=.48, p=.002)$. The correlation coefficient between the RSS and the WMSS posttest indicated a moderate effect size, which suggests that as the RSS increases, the WMSS posttest also tends to increase. Increases in self-esteem also led to increases in weight management self-efficacy. Results suggest that self-esteem plays a key role in the process of increasing weight management self-efficacy. Health professionals should consider addressing self-esteem when conducting health interventions to improve outcomes.

\section{Summary of the Results}

Means, standard deviations, frequencies, and percentages were used for descriptive statistics. The variables that were analyzed were weight management self-efficacy, body image, and self-esteem. The observations for Body Image ranged from 5 to 9 , with an average of $6.55(S D=1.30)$. The observations for RSS ranged from 16 to 40 , with an average of $31.82(S D=5.81)$. The 
observations for the WMSS pretest ranged from 20 to 40, with an average of 27.30 (SD $=4.40)$. The observations for WMSS posttest ranged from 23 to 40 , with an average of $32.12(S D=4.91)$. In the nutrition portion of the WMSS, the pretest ranged from 10 to 20 , with an average of $14.43(S D=2.47)$. The posttest ranged from 10 to 20 , with an average of $17.35(S D=2.68)$. In the physical exercise portion of the WMSS, the pretest ranged from 9 to 20 , with an average of $12.88(S D=2.70)$. The posttest ranged from 5 to 20 , with an average of $14.78(S D=3.49)$. The summary of the results is found in Table 2 .

Table 2. Summary Statistics Table for Interval and Ratio Variables.

$\begin{array}{llllllll}\text { Variable } & M & S D & n & \text { Min. } & \text { Max. } & \text { Skewness } & \text { Kurtosis } \\ \text { Age } & 46.58 & 10.57 & 40 & 22.00 & 64.00 & -0.45 & -0.54 \\ \text { BMI } & 37.16 & 6.90 & 40 & 30.00 & 51.90 & 0.86 & -0.61 \\ \text { BSS } & 6.55 & 1.30 & 40 & 5.00 & 9.00 & 0.17 & -1.06 \\ \text { RSS } & 31.82 & 5.81 & 40 & 16.00 & 40.00 & -0.74 & 0.04 \\ \text { WMSS pretest } & 27.30 & 4.40 & 40 & 20.00 & 40.00 & 0.98 & 1.14 \\ \text { WMSS posttest } & 32.12 & 4.91 & 40 & 23.00 & 40.00 & 0.02 & -1.14 \\ \text { Nutrition Self-efficacy pretest } & 14.43 & 2.47 & 40 & 10.00 & 20.00 & 0.53 & 0.32 \\ \text { Nutrition Self-efficacy posttest } & 17.35 & 2.68 & 40 & 10.00 & 20.00 & -0.99 & 0.58 \\ \text { Exercise Self-efficacy pretest } & 12.88 & 2.70 & 40 & 9.00 & 20.00 & 0.90 & 0.61 \\ \text { Exercise Self-efficacy posttest } & 14.78 & 3.49 & 40 & 5.00 & 20.00 & -0.29 & -0.01\end{array}$

\section{DISCUSSION}

Health coaching had a statistically significant and positive effect on weight management self-efficacy (t $(39)=-6.58, p<.001$ ) among the research sample. Additionally, body image and self-esteem affected weight management self-efficacy; positive health coaching outcomes were significant regardless of positive or negative perceived body image. These findings suggest health professionals should consider body image and self-esteem when creating prevention and treatment programs for obesity. These variables can be addressed using coaching strategies to empower individuals to change and believe in their ability to make positive health decisions.

Personalized and practical health coaching sessions could be used to improve weight management self-efficacy in obese adults. ${ }^{22}$ The coaching process effectively increased self-efficacy, and thus, should be considered in health programs that wish to increase self-efficacy in individual and grouped participants representative of the study sample. Also, body image was correlated with weight management self-efficacy during the pretest but not in the posttest, which suggests that the health coaching sessions helped close the gap between perceived body image and weight management self-efficacy. The coaching process seemed to positively affect participant self-efficacy despite their perceived body image. Additionally, there was a statistically significant correlation between self-esteem and weight management self-efficacy. The results suggest that changes in self-efficacy were influenced by self-esteem and, therefore, self-esteem should be considered when creating obesity interventions.

Healthy lifestyle programs that focus on nutrition and exercise should be designed in a way that addresses individual motivation, barriers to change, self-efficacy, self-esteem, and body image. Additional methods are needed to enable and facilitate behavioral change, with realistic ideas and solutions, based on and tailored to the needs of the individual. ${ }^{22,26}$ Self-efficacy, body image, and self-esteem seemed to affect, hinder, encourage, or motivate individuals' lifestyle changes.

Health coaches should modify and tailor the coaching process according to the needs of their clients. Coaches could merge group education sessions with individual coaching sessions for improved results. ${ }^{40}$ Positive effects can be seen when individuals improve weight management self-efficacy. ${ }^{24}$ Self-esteem and body image should be addressed in health education programs because they are important in the behavior change process. Health coaching can help participants identify potential barriers, develop personalized ways to overcome those barriers, improve adherence to an exercise routine and healthy nutritional habits, and improve self-efficacy. ${ }^{21-22,26}$ Health coaching allows individuals to see areas in need of improvement, their potential via their positive qualities and characteristics, and their inner motivation. Incorporating health coaching to address these areas may improve obese individuals' chances for sustained long-term success. ${ }^{23}$

\section{Limitations}

Some limitations were present in the study and might have affected the results. First, random sampling was not used and the researchers did not control all confounding variables, which means that other possible explanations cannot be ruled out. The study was an open trial with a non-randomized convenience sample, which means that variables like observer-expectancy effect may have accounted for the changes in self-efficacy. In addition, future research studies could provide more time and resources in different areas of the Puerto Rican population to analyze the effects of coaching sessions. Increasing the length of time (i.e., more 
than eight weeks) and resources (e.g., more coaches) could improve the program's effectiveness. The limited time frame and limited personnel to assist in the coaching process could have influenced the results of the study. More randomized studies with a longer time frame are needed to understand the long-term effects of health coaching. Also, a larger and wider sample size could improve external validity. The researcher focused on a medium sized group for direct, personalized, and individualized contact with each participant. The small sample size was comprised of volunteers who wanted to improve their health. This small convenience sample, which lacked a control group, was selected due to time constraints and limited personnel. Also, the study was conducted in the metropolitan area. Future interventions could focus on different parts of the island, including the rural and urban areas. Finally, using a mixed methods intervention in future research could provide more insight and depth into how participants perceive the coaching process itself. Specific experiences and details can provide useful feedback that can enhance coaching program effectiveness.

\section{CONCLUSIONS}

The results of this study suggest health educators should consider addressing body image and self-esteem to create effective obesity programs. Health coaches should recognize the importance of personalized and individualized attention, consistent support, and addressing the personal issues that prevent the individual from changing. The use of a personalized and individualized coaching approach tailored to specific needs, desires, and concerns is an effective method to improve self-efficacy. The role of the health coach is to motivate individuals to change and adapt in a viable and realistic way. Psychosocial variables, such as selfesteem and body image, can affect the outcome of change, and this should be considered to promote best practices in the health and wellness field.

Academia should be focusing more on the emotional and psychological effects of body image and self-esteem and its effect on weight management self-efficacy. Many programs and research studies merely focus on exercise and nutrition as solutions to the obesity epidemic and do not analyze and address the underlying problems like lack of self-esteem or a negative body image. If health professionals want to continue to see long-term changes and positive results, other areas need to be studied and addressed. It was clear that implementing a personalized, individualized, and practical coaching rapport with the individuals, combined with health education, lead to positive results.

\section{REFERENCES}

1. Cochrane AJ, Dick B, King NA, Hills AP, Kavanagh DJ. Developing dimensions for a multicomponent multidisciplinary approach to obesity management: a qualitative study. BMC Public Health. 2017;17:1-15. http://doi:10.1186/s12889-0174834-2.

2. Gubbels JS, Mathisen FK, Samdal O, et al. The assessment of ongoing community-based interventions to prevent obesity: lessons learned. BMC Public Health. 2015;15:216. http://doi.org/10.1186/s12889-015-1563-2

3. Galvão da Silva C, Batalha Sena L, Tavares Palmeira Rolim IL, Alves de Sousa S de M, de Lima Sardinha AH. Nursing care for patients with chronic health conditions: an integrative review. Rev Pesqui: Cuid Fundament. 2017;9(2):599-605. http://doi.org/10.9789/2175-5361.2017.v9i2.599-605

4. Uriyoán Colón-Ramos, Cynthia M. Pérez-Cardona, Rafael Monge-Rojas. Socio-demographic, behavioral, and health correlates of nutrition transition dietary indicators in San Juan, Puerto Rico. Rev Panam Salud Pública. 2013;5:330. Retrieved from http://listserv.paho.org/scripts/wa.exe?A2=JPH;26af5d34.1401

5. Mattei J, Tamez M, Ríos-Bedoya CF, Xiao RS, Tucker KL, Rodríguez-Orengo JF. Health conditions and lifestyle risk factors of adults living in Puerto Rico: a cross-sectional study. BMC Public Health. 2018;18(1):491. http://doi.org/10.1186/s12889018-5359-z

6. Nutrition, physical activity and obesity: Data, trends and maps. Cdc.gov. Retrieved from https://nccd.cdc.gov/NPAO_DTM/LocationSummary.aspx?statecode=117; Updated November 13, 2018. Accessed December 20, 2018.

7. El Burai Félix S, Bailey CM, Zahran HS. Asthma prevalence among Hispanic adults in Puerto Rico and Hispanic adults of Puerto Rican descent in the United States - results from two national surveys. J Asthma. 2015;52(1):39. http://doi.org/10.3109/02770903.2014.950427

8. Elías-Boneta AR, Toro MJ, Garcia O, Torres R, Palacios C. High prevalence of overweight and obesity among a representative sample of Puerto Rican children. BMC Public Health. 2015;15(1):1-8. http://doi.org/10.1186/s12889-015$1549-0$

9. Pérez $\mathrm{C}$, Sánchez H, Ortiz A. Prevalence of overweight and obesity and their cardiometabolic comorbidities in Hispanic adults living in Puerto Rico. J Community Health. 2013;38(6):1140-1146. http://doi.org/10.1007/s10900-013-9726-5 
10. Pérez CM, Soto-Salgado M, Suárez E, Guzmán M, Ortiz AP. High prevalence of diabetes and prediabetes and their coexistence with cardiovascular risk factors in a Hispanic community. J Immigr Minor Health. 2015;17(4):1002-1009. http://doi.org/10.1007/s10903-014-0025-8

11. Torres R, Santos E, Orraca L, Elias A, Palacios C. Diet quality, social determinants, and weight status in Puerto Rican children aged 12 years. J Acad Nutr Diet. 2014;114:1230-1235. http://doi.org/10.1016/j.jand.2014.01.011

12. Salgado-Montilla J, Soto Salgado M, Surillo Trautmann B, Sánchez-Ortiz R, Irizarry-Ramírez M. Association of serum lipid levels and prostate cancer severity among Hispanic Puerto Rican men. Lipids Health Dis. 2015;14:111. http://doi.org/10.1186/s12944-015-0096-0

13. Ashton LM, Hutchesson MJ, Rollo ME, Morgan PJ, Thompson DI, Collins CE. Young adult males' motivators and perceived barriers towards eating healthily and being active: a qualitative study. Int J Behav Nutr Phys Act. 2015;12:1-10. http://doi.org/10.1186/s12966-015-0257-6

14. Burmeister JM, Taylor MB, Rossi J, Kiefner-Burmeister A, Borushok J, Carels RA. Reducing obesity stigma via a brief documentary film: A randomized trial. Stigma Health. 2017;2(1):43-52. http://doi.org/10.1037/sah0000040

15. Farhat $T$, lannotti RJ, Summersett-Ringgold F. Weight, weight perceptions, and health-related quality of life among a national sample of US girls. J Dev Behav Pediatr. 2015;36(5):313-323. http://doi.org/10.1097/DBP.0000000000000172

16. Phelan SM, Burgess DJ, Puhl R, et al. The adverse effect of weight stigma on the well-being of medical students with overweight or obesity: Findings from a national survey. J Gen Intern Med. 2015;30(9):12511258. http://doi.org/10.1007/s11606-015-3266-x

17. Edmonds SE, Zieff SG. Bearing bodies: Physical activity, obesity stigma, and sexuality in the bear community. Sociol Sport J. 2015;32(4):415-435. http://doi.org/10.1123/ssj.2014-0166

18. King EB, Rogelberg SG, Hebl MR, et al. Waistlines and ratings of executives: Does executive status overcome obesity stigma? Hum Resour Manage. 2016;55(2):283-300. http://doi.org/10.1002/hrm.21667

19. Stonerock GL, Blumenthal JA. Role of counseling to promote adherence in healthy lifestyle medicine: Strategies to improve exercise adherence and enhance physical activity. Prog Cardiovasc Dis. 2017;59:455-462. http://doi.org/10.1016/j.pcad.2016.09.003

20. Hill B, Richardson B, Skouteris H. Do we know how to design effective health coaching interventions: A systematic review of the state of the literature. Am J Health Promot. 2015;29(5):e158-e168. http://doi.org/10.4278/ajhp.130510-LIT-238

21. Sforzo GA, Kaye MP, Todorova I, et al. Compendium of the health and wellness coaching literature. Am J Lifestyle Med. 2018;12(6):436. http://doi.org/ 10.1177/1559827617708562

22. Kivelä K, Elo S, Kyngäs H, Kääriäinen M. Review: The effects of health coaching on adult patients with chronic diseases: $A$ systematic review. Patient Educ Couns. 2014;97:147-157. http://doi.org/10.1016/j.pec.2014.07.026

23. Clark MM, Bradley KL, Jenkins SM, et al. Improvements in health behaviors, eating self-efficacy, and goal-setting skills following participation in wellness coaching. Am J Health Promot. 2016;30(6):458-464. http://doi.org/10.4278/ajhp.140627QUAL-304

24. Kozica SL, Lombard CB, Ilic D, Ng S, Harrison CL, Teede HJ. Acceptability of delivery modes for lifestyle advice in a large scale randomised controlled obesity prevention trial. BMC Public Health. 2015;15:699. http://doi.org/10.1186/s12889-0151995-8

25. Stelter R, Andersen V. Coaching for health and lifestyle change: Theory and guidelines for interacting and reflecting with women about their challenges and aspirations. Int Coaching Psychol Rev. 2018;13(1):61-71. https://www.bps.org.uk/

26. Wagner KA, Braun E, Armah SM, et al. Dietary intervention for glucose tolerance in teens (DIG IT): Protocol of a randomized controlled trial using health coaching to prevent youth-onset type 2 diabetes. Contemp Clin Trials. 2017;53:171177. http://doi.org/10.1016/j.cct.2016.12.018

27. Bonsaksen T, Fagermoen MS, Lerdal A. Trajectories of self-efficacy in persons with chronic illness: An explorative longitudinal study. Psychol Health. 2014;29(3):350-364. http://doi.org/10.1080/08870446.2013.856432

28. Bandura, A. Self-efficacy: Toward a unifying theory of behavioral change. Psychol. Rev. 1977;84(2):191-215, 1977. Retrieved from http://dradamvolungis. files.wordpress.com/2011/06/self-efficacy-unifying-theory-of-behavioral-changebandura-1977.pdf

29. Pajares, Frank, and Timothy C. Urdan. Self-efficacy Beliefs of Adolescents. Greenwich, Conn: IAP - Information Age Pub., Inc, 2006. Print.

30. Ickes MJ, McMullen J, Pflug C, Westgate PM. Impact of a university-based program on obese college students' physical activity behaviors, attitudes, and self-efficacy. Am J Health Educ. 2016;47(1):4755. http://doi.org/10.1080/19325037.2015.1111178

31. McGloin H, Timmins F, Coates V, Boore J. A case study approach to the examination of a telephone-based health coaching intervention in facilitating behaviour change for adults with Type 2 diabetes. J Clin Nurs. 2015;24(9-10):1246-1257. http://doi.org/10.1111/jocn.12692 
32. About Adult BMl. Cdc.gov. https://www.cdc.gov/healthyweight/assessing/bmi/adult_bmi/index.html; Updated August 29, 2017. Accessed December 20, 2018.

33. Jerusalem M, Schwarzer R. Self-efficacy as a resource factor in stress appraisal processes. In: Schwarzer R, ed. SelfEfficacy: Thought Control of Action. Washington, DC: Hemisphere Publishing Corp; 1992:195-213.

34. Schwarzer, R., \& Renner, B. (n.d.). Health-specific self-efficacy scales. Retrieved from http://userpage.fuberlin.de/\%7Ehealth/healself.pdf

35. Martín-Albo J, Núñez JL, Navarro JG, Grijalvo F, Navascués V. The Rosenberg Self-Esteem Scale: Translation and validation in university students. Span J Psychol. 2007;10(2):458-467. http://doi.org/10.1017/S1138741600006727

36. Stunkard AJ, Sorensen T, Schulsinger F. Use of the Danish adoption register for the study of obesity and thinness. Res Publ Assoc Res Nerv Ment Dis. 1983;60:115-120.

37. Ralph-Nearman C, Filik R. New body scales reveal body dissatisfaction, thin-ideal, and muscularity-ideal in males. Am J Mens Health. 2018;12(4):740. https://doi-org.proxy1.ncu.edu/10.1177/1557988318763516

38. Rosenberg, M. (1965). Society and the adolescent self-image. Princeton, NJ: Princeton University Press.

39. Nemcek D., Kracek S., \& Perackova J. Rosenberg Self-Esteem Scale analyses among elite and competitive athletes, recreational athletes and inactive individuals. J Phys Educ Sport. 2017;17:23052310. http://doi.org/10.7752/jpes.2017.s5249

40. Bezner JR, Franklin K, Lloyd L, Crixell S. Health behaviour change coaching in physical therapy: improving physical fitness and related psychological constructs of employees in a university setting. Eur J Physiother. 2017;19:1-2. http://doi.org.proxy1.ncu.edu/10.1080/21679169.2017.1381311 